Article

\title{
An Application of Spectral Kurtosis to Separate Hybrid Power Quality Events
}

\author{
Juan José González de la Rosa ${ }^{1,2, *}$, José María Sierra-Fernández ${ }^{1,2}$, \\ José Carlos Palomares-Salas ${ }^{1,2}$, Agustín Agüera-Pérez ${ }^{1,2}$ and Álvaro Jiménez Montero ${ }^{1,2}$ \\ ${ }^{1}$ Research Group Plan Andaluz Investigación Desarrollo Tecnológico e Innovación-Tecnologías de la \\ Información y Comunicación-168 (PAIDI-TIC-168): Computational Instrumentation and Industrial \\ Electronics (ICEI), E-11202 Algeciras, Spain; E-Mails: josemaria.sierra@uca.es (J.M.S.-F.); \\ josecarlos.palomares@uca.es (J.C.P.-S.); agustin.aguera@uca.es (A.A.-P.); \\ alvaro.montero@uca.es (A.J.M.) \\ ${ }^{2}$ University of Cádiz, Area of Electronics, Polytechnic School of Algeciras, Av. Ramón Puyol, S/N, \\ E-11202 Algeciras, Spain \\ * Author to whom correspondence should be addressed; E-Mail: juanjose.delarosa@uca.es; \\ Tel.: +34-956-028-069; Fax: +34-956-028-001.
}

Academic Editor: Thorsten Staake

Received: 1 July 2015 / Accepted: 26 August 2015 / Published: 9 September 2015

\begin{abstract}
For the development of the future smart grid, the detection of power quality events is a key issue for the power system monitoring. Voltage sags, swells, harmonics (variations) and interruptions, which produce large losses for commercial and industrial consumers, are the main events to be considered due to the sensitivity of equipment to these electrical anomalies. The steady-state events are even more frequently accompanied by transients, the discrimination and localization being far more exigent and requiring advanced signal separating tools to be incorporated in the measurement equipment. This paper shows the event detection performance of the spectral kurtosis as a signal separating tool in the frequency domain. The disturbances under test are hybrid signals resulting from the coupling between amplitude defects and non-desired higher frequencies. Being a fourth-order spectrum, the kurtosis is confirmed as a noise-resistant tool that enhances impulsiveness, therefore characterizing the electrical anomalies. In the beginning of the analysis, the voltage sag is established as a reference; then, the disturbances (oscillatory transients and harmonics) are coupled at the starting and ending instants of the sag, resulting in complex hybrid events. The results show that the spectral kurtosis enhances the detection
\end{abstract}


of both types of events (steady state and transients), which are outlined in a bump shape in the fourth-order frequency pattern and centered in the main carrier frequency. Indeed, while the oscillatory transients are associated with softer and lower-amplitude peaks, the harmonics correspond to crisper and higher ones. As these mixed electrical faults are very common in the actual power grid, the article postulates the higher-order spectra to be implemented in prospective online measurement instruments.

Keywords: harmonic distortion; higher-order statistics; mixed PQ(power quality) events; oscillatory transients; power quality; spectral kurtosis

\section{Introduction}

With the uncontrolled growth of society, the consequent energy demand forces drastically adapting the power grid topology. Examples of this change are electric and hybrid cars, which suppose important loads in the power system, and nowadays, they are widely introduced [1,2].

In order to protect the environment, big fossil fuel power plants are being replaced by small renewable energies generators, which suppose enormous implications for the environment and for the power grid. A big synchronous power generator, as can be found in an average power plant, delivers a perfect sinusoidal waveform. In addition, any wave distortion is compensated and converted into a perfect waveform, and it can correct some grid outbalances by generating or consuming reactive power.

At the same time, the power grid is turning into a meshed system. This configuration allows the distribution system to prevent faults, creating new alternate routes for the power flow. In addition, using the information of power generated and consumed in any node and the grid characteristics, it can be configured to minimize losses. However, this has implications when a disturbance occurs. In a mesh system, many lines are connected to any node. In this situation, when a disturbance takes place at a point, it will leave all of the lines connected to that node, expanding quickly through the power grid.

Evolution of the topology of a high voltage power grid could be seen in [3]; the effects of different topologies in a normal function are explained in [4]; and an idea of the complexity level reached by the power gird is given in [5]. All of these complex network schemes require changes in the control, as seen in [6], which implies more elements in the grid. Any additional element could introduce disturbances in the case of a fault.

Collaterally, the new advantages in electrical power generation and power distribution systems have led to a power quality (PQ) degradation. Equipment, such as transformers, motors or conductors, undergo overload and generate low PQ conditions, due to harmonics, insufficient voltage, oscillatory transients or other disturbances. Due to the shared responsibility of $\mathrm{PQ}$, it is important to monitor the voltage and currents at the connection point and in the low, medium and high voltage grid.

One of the most important PQ disturbances is the sag or voltage dip, explained and regulated in [7]. It consists of a reduction of the amplitude value with a duration from a half cycle to a few minutes. Changes in the amplitude usually occur suddenly and carry coupled disturbances: oscillatory transients and temporal harmonic distortions are common during or after amplitude transitions. All of 
those disturbances are examined and explained carefully by IEEE in [8]. Because the amplitude change in the base waveform is usually higher, coupled disturbances are difficult to detect.

In this frame, this work postulates the spectral kurtosis (SK) as a computing tool to detect and characterize transient disturbances that are coupled to steady-state sags. The capabilities of higher-order statistics (HOS) to enhance impulsiveness (non-Gaussian behavior) have been formerly proven in some fields and areas of science and technology [9-16]. More precisely, in the field of the PQ characterization, the research team has developed intelligent methods to detect power quality events, using HOS in the time and frequency domains [17-19].

Regarding direct backgrounds of HOS applications in this field, in the time-domain, several notable works are worthy, e.g., Bollen et al. introduced new statistical features to PQ event detection [20]. In the same direction, $\mathrm{Gu}$ and Bollen [21] found relevant characteristics associated with PQ events in the time and frequency domains. The work by Ribeiro et al. [22] is also remarkable, which extracted new time-domain features based on cumulants. The same authors performed the classification of single and multiples disturbances using HOS in the time domain and Bayes' theory-based techniques [23]. HOS techniques and estimators have also been implemented to specifically detect sags and swells [24]. The categorization of PQ anomalies had been formerly performed by Nezih and Ece in [25], where they proved that HOS and quadratic classifiers improve the second order-based methods. The most aligned work is the one developed by Liu et al. in [26], where the researchers extracted a rich set of higher-order features related to the analysis as different types of PQ events using spectral kurtosis.

The paper is structured as follows. Firstly, the SK procedure is explained in Section 2, then coupled disturbances are examined using the SK analysis in the Section 3. Finally, conclusions are drawn in Section 4.

\section{The Spectral Kurtosis: Procedure of Analysis}

The SK is a fourth-order spectrum that deals with the peakedness of the probability density function associated with a measurement data series. From a practical point of view, the traditional power spectrum does not reflect these impulsive features of the time series, which indeed constitute the key attributes of PQ events. For that reason, the SK pattern should reflect the transient characteristics associated with the frequencies under test [12].

The procedure of computation is explained as follows. The time domain register is chopped into realizations or segments overlapped by $50 \%$. This means that the second half of a time realization is the first half of the next realization. Then, the FFT of each realization is calculated and stored in a matrix, where every new FFT is added in a new row. With the data presented this way, Equation (1) represents the estimator involved in the SK computation:

$$
\hat{G}_{2, X}^{N, M}(m)=\frac{M}{M-1}\left[\frac{(M+1) \sum_{i=1}^{M}\left|X_{N}^{i}(m)\right|^{4}}{\left(\sum_{i=1}^{M}\left|X_{N}^{i}(m)\right|^{2}\right)^{2}}-2\right]
$$

where $\mathbf{X}$ is the FFT matrix, in which the upper index $i$ means the row (realization) and the index $m$ is associated with the column (frequency). $M$ realizations with $\mathrm{N}$ points have been considered. The final SK vector has the same points as the FFT vector; equal to $N / 2$. The more realizations are involved in 
the calculation, the more precise is this procedure. However, the computational load increases with the size and the number of realizations, so a correct size and number of points must be indicated for the realizations to achieve suitable resolution and precision, but with an acceptable computational burden; in this case, 1000 realizations of $0.1 \mathrm{~s}$ each, using a sampling frequency of $20,000 \mathrm{~Hz}$ for all analysis. This means an SK vector of 1000 points and a maximum frequency of 10,000 Hz.

As said formerly, 1000 realizations of $0.1 \mathrm{~s}$ are needed; however, each register has only a 1-s length. This problem is overcome using a sliding version for the SK estimator. The spectral data are accumulated for $100 \mathrm{~s}$ before the SK could be calculated.

Hereinafter, the results are presented, dividing them into groups corresponding to different types of electrical perturbations.

\section{Coupled Disturbances}

In this section, an evolution to a coupled signal will be done. Firstly, the base response for a healthy signal will be set. Then, the sag will be examined alone, and finally, a sag with an oscillatory transient and harmonic distortion coupled is studied.

Firstly, and with the goal of getting a reference, the analysis of a healthy signal is performed (shown in Figure 1). The healthy condition is stated as a sinusoid of unitary amplitude. It will be the base amplitude assumed throughout the work.

With the frequency pattern characterization of this ideal signal, the anomalies associated with the disturbances are detected more easily. Synthetics are used in order to control all of the signal parameters, allowing in this way a correct comparison among them. In order to introduce real-life conditions, additive Gaussian noise with a standard deviation $(\sigma)$ of 0.05 is added to all signals.

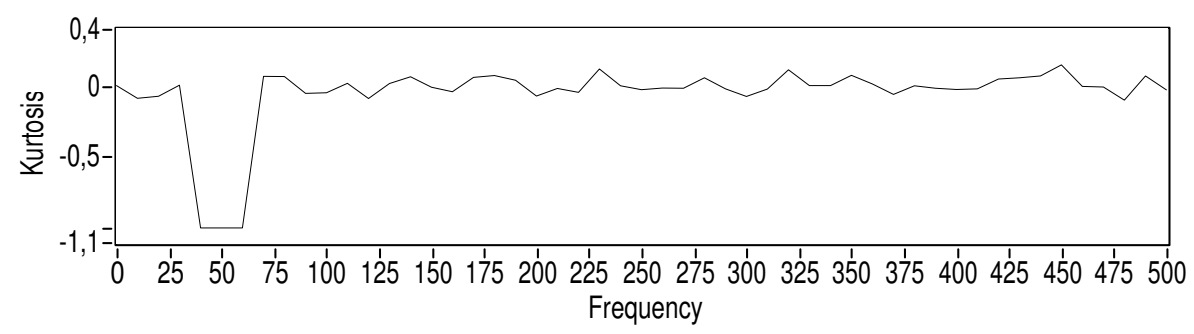

Figure 1. Spectral kurtosis (SK) analysis over a healthy signal. SK results corresponding to a $50-\mathrm{Hz}$ sinusoid with additive Gaussian noise with $(\sigma=0.05)$, swept from 0 to $500 \mathrm{~Hz}$.

Signals have been synthesized with the same properties as described in the SK definition, i.e., 20,000 samples per second. That means a maximum frequency of $10,000 \mathrm{~Hz}$ for the SK analysis. However, the graph only shows up to $500 \mathrm{~Hz}$. In the graph, the same response from 100 to $500 \mathrm{~Hz}$ can be seen. It continues equally for the rest of the frequencies. As no new information could be extracted from frequencies higher than $500 \mathrm{~Hz}$, only frequencies under that value are shown, obtaining in this way a more detailed representation. Hereinafter, this is performed for all experiments, due to all frequencies involved in the considered disturbances being lower than $500 \mathrm{~Hz}$.

In Figure 1, the same pattern is outlined for almost all of the frequencies. These are small variations around the zero, with a maximum deviation of 0.2 . This value is related to the additive noise, which 
is rejected by the SK. In the lower frequency band of the graph, a -1-valued peak appears, centered at a frequency of $50 \mathrm{~Hz}$. With a discrete spectral resolution of $10 \mathrm{~Hz}$, the -1 value copes with the frequencies of 40 and $60 \mathrm{~Hz}$. This zone is associated with the power signal frequency and indicates the feature associated with a constant amplitude signal.

Being a higher-order spectrum, the SK is postulated as a noise-resistant analysis tool. This property is tested studying different noise contamination levels. Table 1 shows the kurtosis values for the base frequency $(50 \mathrm{~Hz})$ and maximum variation around zero for the rest of the spectrum for Gaussian additive noise with $\sigma$ from 0.05 to 0.80 . If a $50-\mathrm{Hz}$ kurtosis value is examining, no important change could be observed. As noise contamination increases, kurtosis takes values a litter higher, from -0.99998 to -0.9962 . However, in this range, all could be considered -1 , with an error lower than $1 \%$. In relation to the maximum variation around zero, there are no relations with the noise contamination. Different values could be seen for different contamination levels, with a maximum value of 0.345210 and a minimum value of 0.206976 . As a test of the randomness of these values, 1000 analyses with the $\sigma=0.05$ condition have been performed. Each single analysis has returned a different maximum variation value around zero, the higher one being 0.417784 (only in two analyses) and the lower one 0.177371 (in some of them). The average value obtained in this experiment is 0.25 .

Table 1. SK analysis results for a healthy signal with different noise contamination levels.

\begin{tabular}{ccc}
\hline Noise level $(\sigma)$ & $\mathbf{5 0 - H z}$ Peak value & Maximum variation around zero \\
\hline 0.05 & -0.999986 & 0.250188 \\
0.10 & -0.999937 & 0.209871 \\
0.15 & -0.999866 & 0.251025 \\
0.20 & -0.999761 & 0.206976 \\
0.25 & -0.999599 & 0.234948 \\
0.30 & -0.999480 & 0.210391 \\
0.35 & -0.999311 & 0.259246 \\
0.40 & -0.999089 & 0.213317 \\
0.50 & -0.998368 & 0.263187 \\
0.60 & -0.997846 & 0.345210 \\
0.70 & -0.997300 & 0.216441 \\
0.80 & -0.996223 & 0.238456 \\
\hline
\end{tabular}

The SK analysis associated with a non-defective state is examined. Now, the sag is introduced in the signal, and the differences in the SK pattern are analyzed, in order to establish the base analysis condition for isolated sags, so as to be able to study coupled disturbances. Signals are presented without Gaussian additive noise, with the objective of getting a cleaner representation. In Figure 2, the sag disturbance is represented, and Figure 3 depicts its associated SK analysis. 


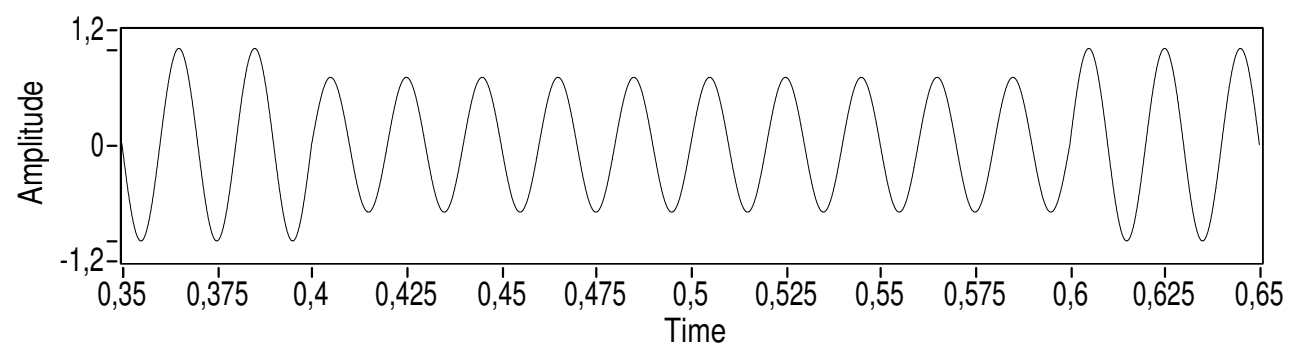

Figure 2. Sag. Time series of a sag with a depth of $30 \%$ and a duration of 10 cycles.

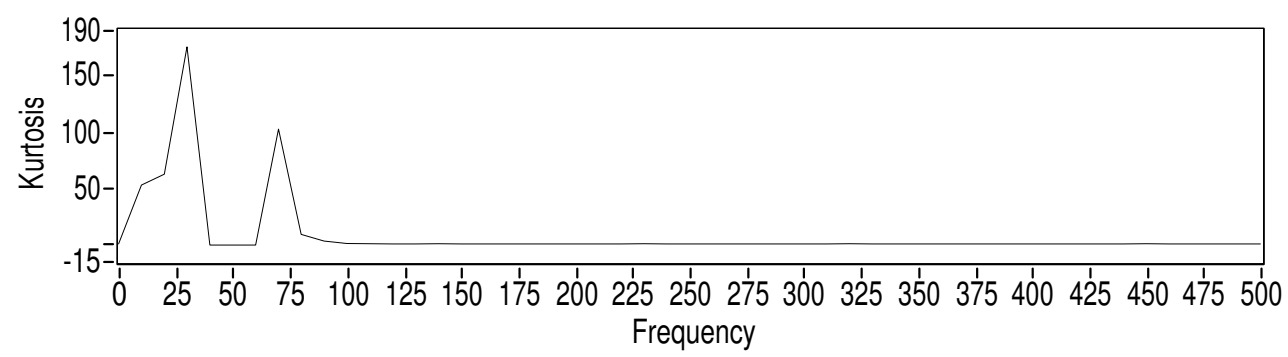

Figure 3. SK analysis over a sag. SK analysis shown from 0 to $500 \mathrm{~Hz}$ of a sag with Gaussian additive noise with $\sigma=0.05$.

As said above, a sag is a reduction in the amplitude of the base waveform. In this experiment, the amplitude has been reduced by $30 \%$, with crisp transitions.

In relation to the SK analysis, there are some changes. First, the vertical axis limits have been changed. The graph in Figure 1 shows the kurtosis values from -1.1 to 0.4 . Now, it spreads from -15 to 190 .

From $100 \mathrm{~Hz}$ onwards, the kurtosis response is constant, close to zero. If a zoom is made over this area, the same response is observed. However, with this vertical scale, it seems a constant value. The main change occurs in frequencies under $100 \mathrm{~Hz}$. Two peaks appear, one of them before $50 \mathrm{~Hz}$ and the other one after this frequency component. This separation is occasioned by the same -1 peak at $50 \mathrm{~Hz}$, seen in the previous analysis. As an important length is computed, even with the momentary change of amplitude, $50 \mathrm{~Hz}$ is still considered as a constant amplitude. That is represented with the -1 value at $50 \mathrm{~Hz}$. Here, the -1 kurtosis value could not be seen due to the vertical scale, but it shows exactly the same value as in the healthy signal. When the amplitude of a single frequency changes suddenly, the surrounding frequencies are affected, as well. That is the situation now. A kurtosis increase for frequencies around $50 \mathrm{~Hz}$ means an increase in the variability of these components. They do not reach important amplitudes, however, as they do not show any amplitude before; this means important amplitude variations.

Different sag conditions have been tested in the next experiment, keeping the noise contamination constant in $\sigma=0.05$. Depths from 10 to $90 \%$ are settled, considering durations from 5 to 20 cycles. In each signal, kurtosis values for frequencies of 30,50, 70, 100 and $150 \mathrm{~Hz}$ are taken. These frequencies are related to the first peak, the base frequency, the second peak, a near frequency in which the system is slightly altered and a far frequency in which the system is almost unaffected. All information related to those experiments is shown in the Table 2. 
Table 2. SK analysis results for sag disturbance with different depths and lengths.

\begin{tabular}{|c|c|c|c|c|c|c|}
\hline \multirow{2}{*}{$\begin{array}{c}\text { Sag deep } \\
(\%)\end{array}$} & \multirow{2}{*}{$\begin{array}{c}\text { Sag length } \\
\text { (Cycles) }\end{array}$} & \multicolumn{5}{|c|}{ Kurtosis } \\
\hline & & $30 \mathrm{~Hz}$ & $50 \mathrm{~Hz}$ & $70 \mathrm{~Hz}$ & $100 \mathrm{~Hz}$ & $150 \mathrm{~Hz}$ \\
\hline 10 & 5 & 8794 & -0.999 & 4842 & 0.067 & 0.126 \\
\hline 10 & 10 & 9044 & -0.999 & 2,643 & -0.053 & 0.074 \\
\hline 10 & 20 & 8175 & -0.999 & 2592 & -0.047 & 0.084 \\
\hline 20 & 5 & 81,769 & -0.999 & 33,701 & 0.051 & 0.127 \\
\hline 20 & 10 & 81,857 & -0.999 & 35,095 & -0.005 & 0.077 \\
\hline 20 & 20 & 77,130 & -0.998 & 27,154 & 0.193 & 0.080 \\
\hline 30 & 5 & 173,911 & -0.999 & 98,029 & 1066 & 0.146 \\
\hline 30 & 10 & 181,324 & -0.999 & 102,501 & 1099 & 0.088 \\
\hline 30 & 20 & 178,341 & -0.998 & 95,583 & 1160 & 0.084 \\
\hline 40 & 5 & 256,598 & -0.999 & 174,311 & 2660 & 0.185 \\
\hline 40 & 10 & 261,075 & -0.998 & 172,657 & 1127 & 0.242 \\
\hline 40 & 20 & 260,819 & -0.996 & 168,985 & 2234 & 0.108 \\
\hline 50 & 5 & 319,816 & -0.999 & 233,250 & 6121 & 0.199 \\
\hline 50 & 10 & 329,233 & -0.997 & 237,407 & 3534 & 0.208 \\
\hline 50 & 20 & 323,410 & -0.995 & 244,310 & 5246 & 0.151 \\
\hline 60 & 5 & 361,178 & -0.998 & 289,918 & 11,350 & 0.305 \\
\hline 60 & 10 & 368,614 & -0.997 & 288,906 & 9536 & 0.237 \\
\hline 60 & 20 & 366,564 & -0.994 & 290,237 & 11,325 & 0.242 \\
\hline 70 & 5 & 392,615 & -0.998 & 329,945 & 16,659 & 0.334 \\
\hline 70 & 10 & 398,564 & -0.996 & 326,260 & 16,300 & 1,121 \\
\hline 70 & 20 & 396,867 & -0.993 & 327,962 & 17,368 & 0.366 \\
\hline 80 & 5 & 415,655 & -0.998 & 360,687 & 29,481 & 0.828 \\
\hline 80 & 10 & 418,429 & -0.996 & 357,976 & 27,213 & 0.890 \\
\hline 80 & 20 & 418,173 & -0.992 & 359,114 & 26,520 & 0.769 \\
\hline 90 & 5 & 431,010 & -0.998 & 386,573 & 38,483 & 1488 \\
\hline 90 & 10 & 433,368 & -0.996 & 384,002 & 42,927 & 1236 \\
\hline 90 & 20 & 433,365 & -0.992 & 383,941 & 40,564 & 0.830 \\
\hline
\end{tabular}

As indicated above, at $50 \mathrm{~Hz}$, the peaks could be considered -1, even in the higher depth and length conditions. Values in this table are truncated, not rounded.

The durations of the disturbances have no effect on the kurtosis values, keeping the same value for each depth. In relation to the depth, a higher one involves a higher alteration of the waveform. SK detects frequency variability, showing higher values of kurtosis as sag depth increases.

In Table 2 and in Figure 3, it is seen that the 30-Hz frequency peak has a kurtosis value higher than the peak at $70 \mathrm{~Hz}$. In a visual examination of all experiments, $100 \mathrm{~Hz}$ seems not to be altered by the sag disturbance. However, a detailed analysis reveals that the kurtosis value is increased when peaks reach 
important values. Nevertheless, the value associated with $150 \mathrm{~Hz}$ is hardly altered, even in the higher depth and length conditions.

Up to now, the base condition of a healthy signal and a sag disturbance alone has been intensely studied. Sag will be coupled to some disturbances. SK analysis will be studied in all of the situations, searching the characteristics of all disturbances introduced, with the goal of differentiating all of them. In the rest of the experiments, a depth of $30 \%$ and a length of 10 cycles have been selected.

Then, the oscillatory transient will be coupled to the sag. It consists of oscillations around the normal waveform value, with a crisp start and the exponential decay of its amplitude. The signal shown in Figure 4 is composed of the sag indicated before and the oscillatory transient with oscillations of 200 $\mathrm{Hz}$, a duration of four cycles of the power waveform and an initial amplitude of 0.2 . It has one oscillatory transient at the sag start and another at the sag end. As in previous graphs, this is shown without noise, in order to observe the disturbance better. In Figure 5, the SK analysis of the coupled signal is shown.

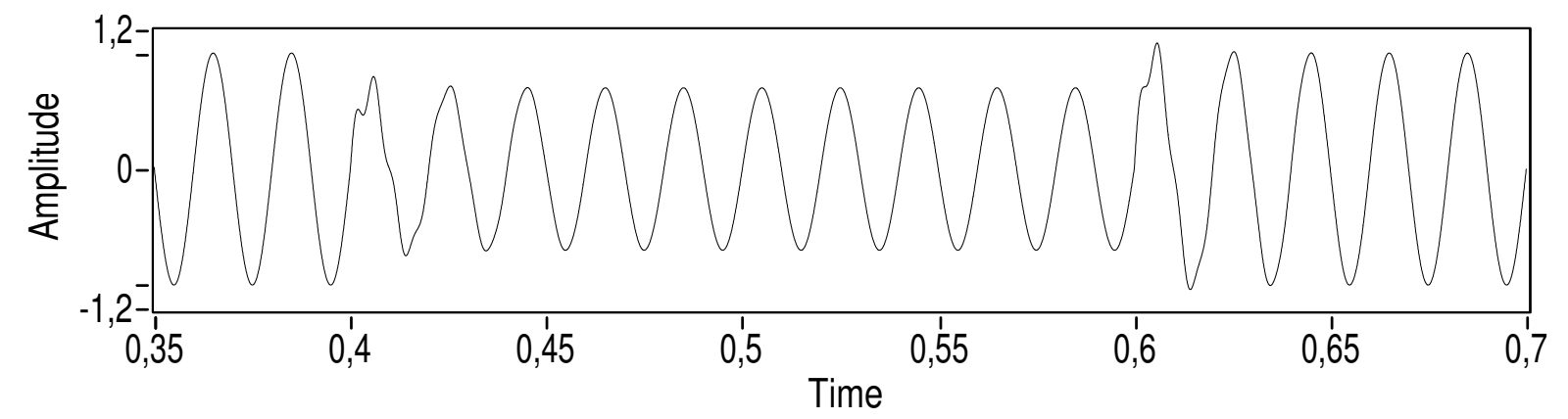

Figure 4. Sag with a coupled oscillatory transient. Time series of a sag with a depth of $30 \%$ and a duration of 10 cycles, with an oscillatory transient with a maximum amplitude of a 0.2 , a duration of four cycles of the power signal and a frequency of $200 \mathrm{~Hz}$.

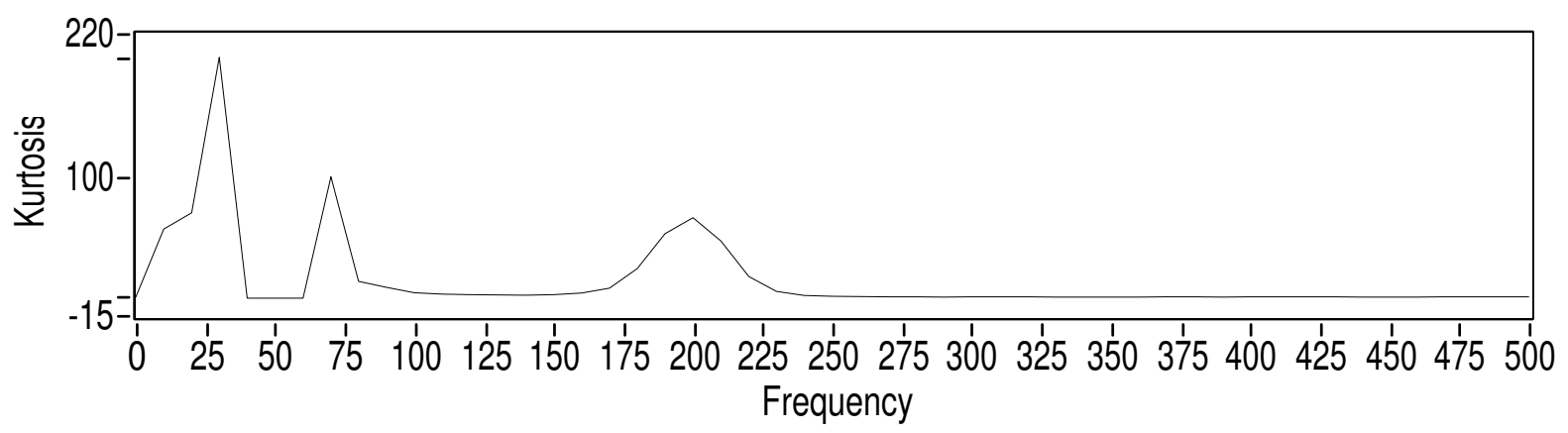

Figure 5. SK analysis over a sag with a coupled oscillatory transient. SK results shown from 0 to $500 \mathrm{~Hz}$ of a sag with an coupled oscillatory transient, with Gaussian additive noise with $\sigma=0.05$.

The main frequency introduced with the oscillatory transient is $200 \mathrm{~Hz}$. As mentioned above, the sags do not alter the frequency over $150 \mathrm{~Hz}$. At first glance, a new element could be seen. In the zone of the graph corresponding to lower frequencies, the same peaks as in the sag situation could be seen. Thus, the sags are detected the same as if this were the only disturbance. However, another peak appears around $200 \mathrm{~Hz}$, which is the oscillatory frequency. This last peak is associated with the oscillatory transient. 
As in the sag case, an oscillatory transient supposes an amplitude variation. However, on the contrary, it does not show a constant amplitude during all of the analyzed vectors, so it does not have a -1 value in the central frequency. The sudden start in an oscillatory transient creates an increment in the variability of oscillation frequency. In addition, the transient amplitude is continuously decreasing, which increases the variability of the oscillation frequency more. All of this affects the surrounding frequencies, increasing their variability, as well. The result of all of this is the peak shown in the graph, with a maximum value in the central frequency and a soft decreasing of the kurtosis value as the frequency moves away from $200 \mathrm{~Hz}$, symmetrically in both directions.

Some oscillatory transient conditions are considered, keeping the same sag conditions as indicated before. The oscillatory transient amplitudes taken are from 0.15 to 0.3 , and the lengths considered for each amplitude are from two to 10 power signal cycles. As symmetrical peak is returned; only the information of the half peak is taken, being similar on the other side. The kurtosis values for frequencies of 200,210, 220 and $230 \mathrm{~Hz}$ have been taken, and the peak width is measured with the number of points around $200 \mathrm{~Hz}$ that have kurtosis values over three. This can be seen in Table 3 .

Table 3. SK analysis results for a sag with an oscillatory transient coupled with different conditions.

\begin{tabular}{cc|cccc|c}
\hline \multirow{2}{*}{$\begin{array}{c}\text { Transient } \\
\text { amplitude }\end{array}$} & $\begin{array}{c}\text { Transient } \\
\text { length }\end{array}$ & \multicolumn{4}{|c|}{ Kurtosis value } & \multirow{2}{*}{$\begin{array}{c}\text { Peak } \\
\text { width }\end{array}$} \\
\cline { 3 - 6 } & $\mathbf{2 0 0 ~ H z}$ & $\mathbf{2 1 0 ~ H z}$ & $\mathbf{2 2 0 ~ H z}$ & $\mathbf{2 3 0 ~ H z}$ & \\
\hline 0.15 & 2 & 4026 & 3654 & 1519 & 0.446 & 4 \\
0.15 & 4 & 28,877 & 20,731 & 6484 & 1958 & 6 \\
0.15 & 6 & 58,321 & 40,097 & 12,979 & 2461 & 6 \\
0.15 & 10 & 80,057 & 50,000 & 10,989 & 2050 & 6 \\
\hline 0.20 & 2 & 12,743 & 10,804 & 4,665 & 1882 & 6 \\
0.20 & 4 & 62,293 & 49,007 & 12,880 & 3077 & 7 \\
0.20 & 6 & 93,304 & 71,551 & 22,576 & 3445 & 7 \\
0.20 & 10 & 118,725 & 91,339 & 25,939 & 4212 & 8 \\
\hline 0.25 & 2 & 32,125 & 23,639 & 10,084 & 5794 & 11 \\
0.25 & 4 & 99,072 & 82,614 & 35,334 & 10,221 & 11 \\
0.25 & 6 & 140,299 & 117,120 & 45,022 & 11,404 & 12 \\
0.25 & 10 & 150,465 & 132,738 & 56,852 & 11,749 & 13 \\
\hline 0.30 & 2 & 54,755 & 39,571 & 19,989 & 9493 & 13 \\
0.30 & 4 & 148,846 & 117,608 & 53,017 & 17,717 & 13 \\
0.30 & 6 & 167,414 & 152,665 & 78,887 & 23,714 & 14 \\
0.30 & 10 & 169,960 & 166,178 & 83,782 & 23,326 & 13 \\
\hline
\end{tabular}

It is worth remarking about the following issues. Firstly, the length of two cycles reduces the kurtosis value in the oscillation frequency, for all amplitude situations. Simulation creates a sinusoidal wave with the desired length and applies an exponential decrease in the transient amplitude, which ends in zero. The shorter the transients were, the more the amplitude of the first cycle would be reduced. This reduces 
the initial change of the amplitude. Moreover, the variability is related to the change of amplitude during the transient, and as it has a lower extension, the variability is reduced, as well.

This trend continues as length increases. All kurtosis values are higher as the lengths of transients increase. This could be observed in any frequency for any amplitude considered in the table.

As indicated before, the frequencies selected in the table are related to the half peak. The alteration of SK is symmetrical, as can be seen in Figure 5. As the transient amplitude increases, the peak turns softer. This means a higher variation as the frequency moves away from the central one. For the 0.3 amplitude and a 10-cycle length, the kurtosis associated with 200 and $210 \mathrm{~Hz}$ is almost the same, and the next frequency shows half value of them. However, for the same length, taking an amplitude of 0.2 , a change from 200 to $210 \mathrm{~Hz}$ supposes a loss of 23\%, and in the next step, it losses $71 \%$. Higher variations, as seen for the second one, mean a pointier peak. Lower kurtosis variations among near frequencies, as in the first one, create a softer peak, with a rounder shape.

In addition, the higher amplitude means a higher variability itself. At the starting moment, it creates a bigger step, and then, during the exponential decay, a bigger change is observed. Taking the same two situations, with an amplitude of 0.3 and a length of 10 cycles, the maximum value returned is 169.96 . With the same length, and for an amplitude of 0.2 , a peak with a maximum of 118.72 is originated.

The last column shows the width of the peaks, taking a threshold of three. An increase in the length multiplies the kurtosis value associated with each frequency. As frequencies near the end of each peak have very low values, multiplying them does not change the situation very much. This could be observed by the fact that almost the same length is indicated for each amplitude. On the other hand, as the transient amplitude increases, the kurtosis value is incremented, increasing at the same time the peak widths and turning them softer.

The last disturbance considered is the harmonic distortion at the sag start and end. The harmonic selected is the fourth one, which implies a frequency of $200 \mathrm{~Hz}$. This one has been used in order to introduce the same frequency as in the previous test. In the oscillatory transient situation, oscillations starts with a high amplitude, and this decays in an exponential way. On the other hand, the harmonics take the same amplitude during all of the disturbed segment. In Figure 6, the signals with harmonic distortion are shown, and the SK analysis is shown in Figure 7 from 0 to $500 \mathrm{~Hz}$.

The conditions for the sag are the same as in the previous experiments. The same frequency has been introduced; any difference observed in relation to the oscillatory transient are due to harmonic distortion. The oscillatory transient introduced in Figure 4 starts with a high alteration; however, the signal recovers its normal state very quickly. On the other hand, in Figure 6, changes in the signal are at the same level for all of the disturbed segment.

In Figure 7, the same response for frequencies under $100 \mathrm{~Hz}$ as in Figure 5 can be seen. This is related to the sag: as the same conditions have been considered, its response remains equal, as well. There is another peak around $200 \mathrm{~Hz}$, in the same position as in the previous experiment. However, the peak's shape is different. Now, it takes a shorter extension and reaches a higher kurtosis value, resulting in a pointier peak than in the previous situation. 


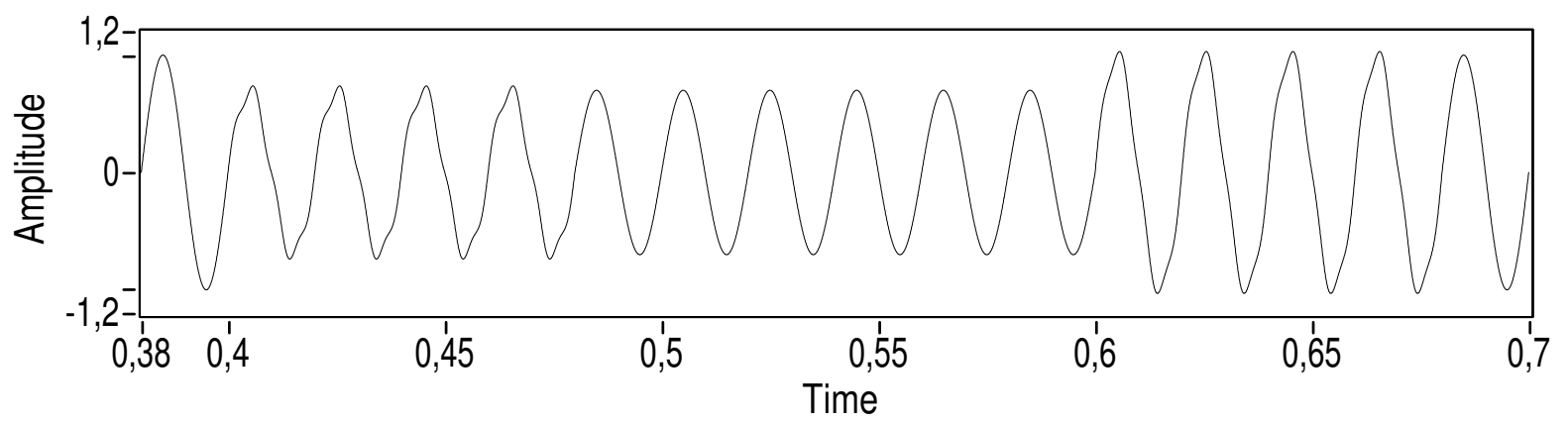

Figure 6. Sag with harmonic distortion. Time series of a sag with a depth of $30 \%$ and a duration of 10 cycles with harmonic distortion with an amplitude of a 0.07 and a duration of four cycles of the power signal. The fourth harmonic has been selected $(200 \mathrm{~Hz})$.

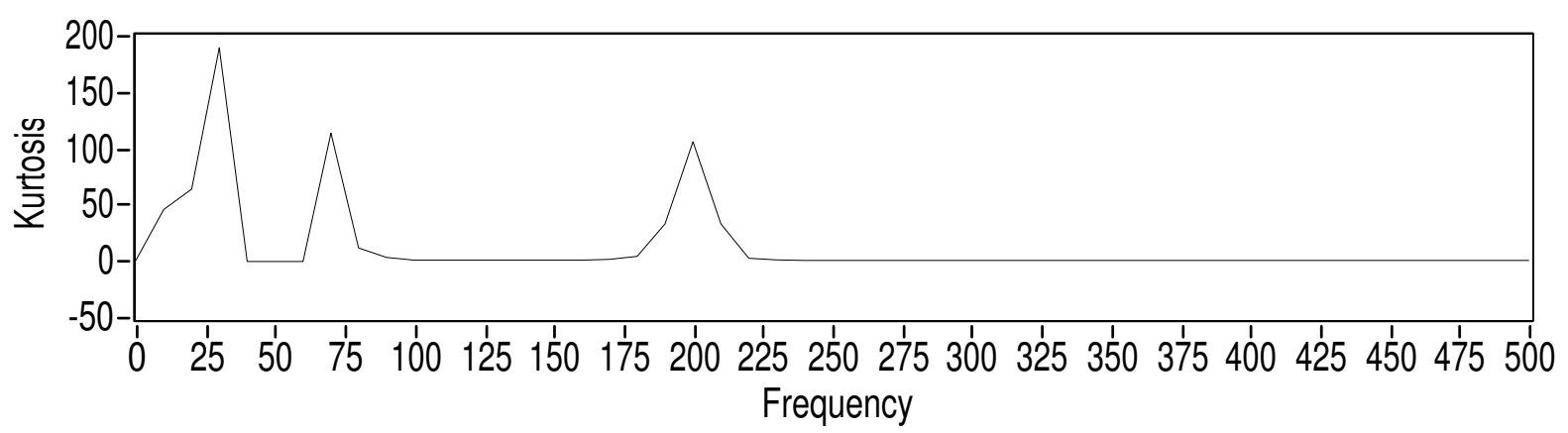

Figure 7. Sag analysis with harmonic distortion. SK results shown from 0 to $500 \mathrm{~Hz}$ of a sag with harmonic distortion, with Gaussian additive noise with $\sigma=0.05$.

As in the oscillatory transient experiment, some harmonic disturbance conditions will be tested, keeping the sag conditions and the harmonic distortion frequency equal. Harmonic distortion amplitudes have been taken from 0.07 to 0.20 , using lengths from one to four cycles of the base waveform. The results of these experiments are shown in Table 4. As in the oscillatory transient situation, the kurtosis value related to each signal has been taken for frequencies of 200, 210, 220 and $230 \mathrm{~Hz}$, and the peak widths have been measured as the number of points with a kurtosis value over three around $200 \mathrm{~Hz}$.

In this experiment, lower extensions and amplitudes have been considered. Even with that, higher kurtosis values are obtained.

Examining Figure 6, the harmonic distortion peak looks pointier than the one related to the oscillatory transient, shown in Figure 4. Comparing the conditions shown in the figures, for the same length, four cycles of the base waveform, harmonic distortion with an amplitude 0.07 creates a three-point peak with a maximum value of 104 , and an oscillatory transient, with an amplitude of 0.20 , creates a seven-point peak with a maximum value of 62 . The peak related to harmonic distortion shows a higher kurtosis value and a lower width than that related to the oscillatory transient.

If the same conditions are used for harmonic distortion and the oscillatory transient, for example an amplitude of 0.20 and a length of four cycles of the base waveform, different results are obtained. Harmonic distortion gives a peak with a maximum value of 261 and a width of nine, and the oscillatory transient returns a peak with a maximum value of 62 and a width of seven. Now, the peak related to harmonic distortion is wider; however, if the ratio between the maximum value and the width is shown, 
the harmonic distortion has $260 / 9=28.9$ and the oscillatory transient $62 / 7=8.8$. This means that the harmonic peak is taller in relation to its width than the one related to the oscillatory transient.

Table 4. SK analysis results for sag with harmonic distortion coupled with different conditions.

\begin{tabular}{|c|c|c|c|c|c|c|}
\hline \multirow{2}{*}{$\begin{array}{l}\text { Harmonics } \\
\text { amplitude }\end{array}$} & \multirow{2}{*}{$\begin{array}{c}\text { Transient } \\
\text { length }\end{array}$} & \multicolumn{4}{|c|}{ Kurtosis value } & \multirow{2}{*}{$\begin{array}{l}\text { Peak } \\
\text { width }\end{array}$} \\
\hline & & $200 \mathrm{~Hz}$ & $210 \mathrm{~Hz}$ & $220 \mathrm{~Hz}$ & $230 \mathrm{~Hz}$ & \\
\hline 0.07 & 1 & 10,048 & 5804 & 3028 & 0.549 & 5 \\
\hline 0.07 & 2 & 28,624 & 18,553 & 2112 & 0.156 & 4 \\
\hline 0.07 & 3 & 58,138 & 25,196 & 2011 & 0.200 & 4 \\
\hline 0.07 & 4 & 104,395 & 34,220 & 1069 & 0.209 & 3 \\
\hline 0.10 & 1 & 32,189 & 25,505 & 11,373 & 2150 & 6 \\
\hline 0.10 & 2 & 64,873 & 44,329 & 6894 & 0.540 & 5 \\
\hline 0.10 & 3 & 116,762 & 68,756 & 6654 & 0.571 & 5 \\
\hline 0.10 & 4 & 169,964 & 72,610 & 4926 & 0.637 & 5 \\
\hline 0.15 & 1 & 84,983 & 72,386 & 41,950 & 9014 & 9 \\
\hline 0.15 & 2 & 127,734 & 89,248 & 20,804 & 1935 & 7 \\
\hline 0.15 & 3 & 174,153 & 126,729 & 23,235 & 2674 & 6 \\
\hline 0.15 & 4 & 236,331 & 129,797 & 13,634 & 1399 & 5 \\
\hline 0.20 & 1 & 149,340 & 135,240 & 84,174 & 27,454 & 8 \\
\hline 0.20 & 2 & 178,761 & 133,981 & 49,134 & 7189 & 9 \\
\hline 0.20 & 3 & 207,419 & 164,963 & 45,999 & 6692 & 8 \\
\hline 0.20 & 4 & 261,294 & 166,906 & 41,016 & 5087 & 9 \\
\hline
\end{tabular}

As indicated before, with the same conditions, the harmonic distortion generates kurtosis responses higher than the oscillatory transients. Taking the conditions of an amplitude of 0.20 and a duration of two cycles, the maximum kurtosis values are 12.7 for the oscillatory transient and 178.7 for the harmonic distortion. This difference is accentuated for a duration for four cycles and the same amplitude, reaching values of 62.3 for the oscillatory transient and 261.3 for the harmonic distortion. If the same maximum value is searched, an amplitude of 0.07 and a duration of three cycles must be used for harmonic distortion, obtaining a value of 58.13, and an amplitude of 0.15 with a duration of six cycles for the oscillatory transient, obtaining a value of 58.32 .

As said before, the peaks associated with harmonic distortion are sharper than the ones related to the oscillatory transients, and now, this will be detected over the kurtosis evolution. First, the kurtosis change from the peak central frequency $(200 \mathrm{~Hz})$ to the next one $(210 \mathrm{~Hz})$ will be examined, for common conditions. These are amplitudes of 0.15 and 0.20 and durations of two and four base signal cycles. In these conditions, the change rates are from $9 \%$ to $30 \%$ for oscillatory transients and from $25 \%$ to $45 \%$ for harmonic distortion. If all simulations are examined, variation rates of $2 \%$ and $37 \%$ are obtained for oscillatory transients, and for harmonics distortion, these take values from $9 \%$ to $67 \%$. Taking common 
conditions or taking all simulations, a higher variation could be observed for harmonic distortion. This higher change implies a crisper peak.

With the goal of considering a real-life situation, we have introduced Figure 8, where a sag is accompanied by a severe harmonic distortion (the same as in Figure 8) in a Gaussian additive noise floor with $\sigma=0.5$. In this case, the measurement bandwidth is wider, taking the whole profit of the data acquisition equipment, connected via a differential probe to the phase voltage A-B. The complete analysis is also shown, where the time domain variance (power variations), skewness (indicator of the waveform symmetry) and the kurtosis (peakedness of the time domain signal) are also presented. The peaks in the SK graph are the same as those in Figure 7. In fact, the amplitude transition is reflected as a spectral leakage around the harmonics. Indeed, the y-axis in the SK graphs accounts for variability, as the kurtosis is an estimator of the peakedness of the probability density function associated with the measurement time series.
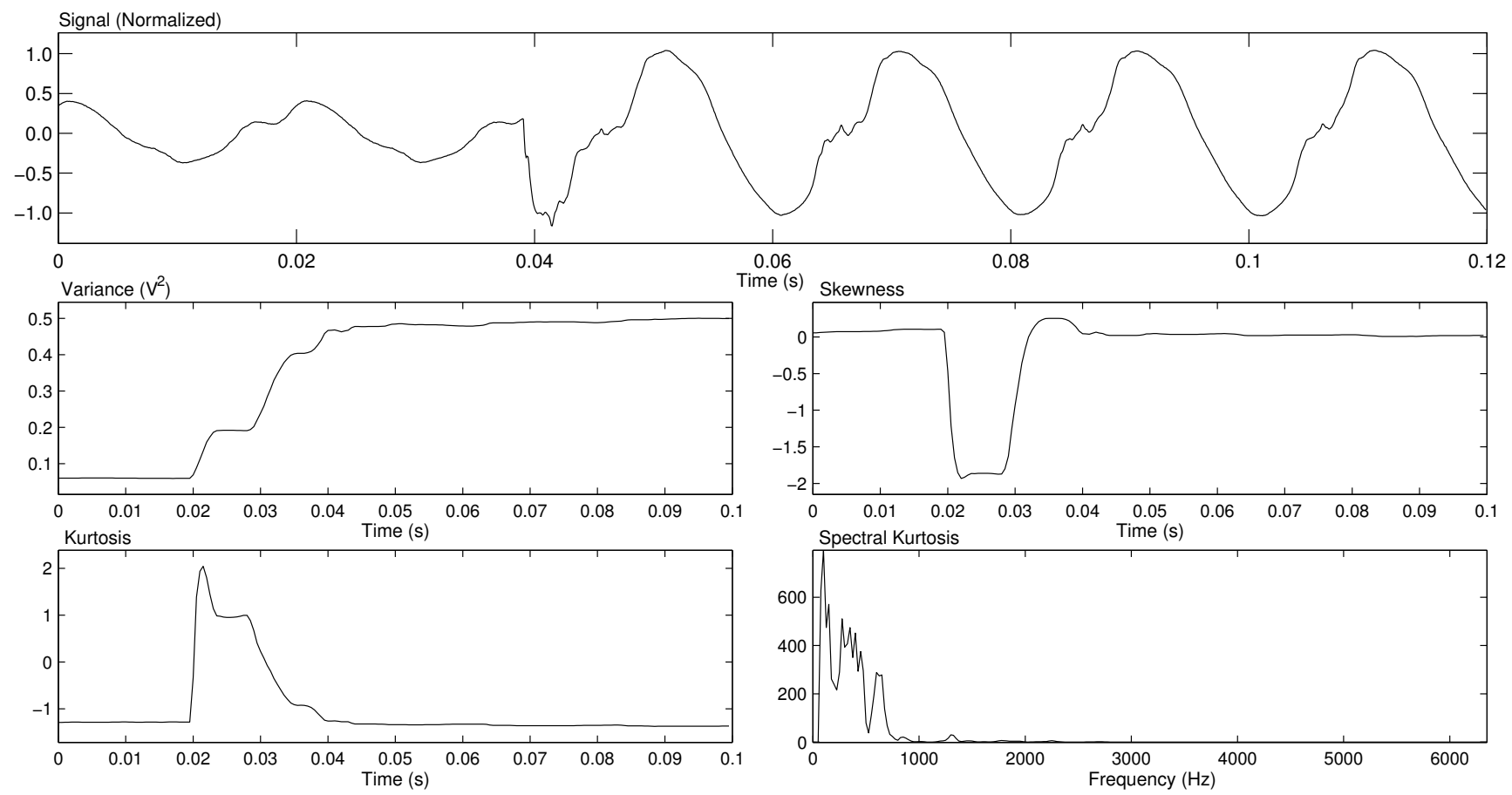

Figure 8. SK analysis over a sag with harmonic distortion, with Gaussian additive noise with $\sigma=0.05$. If harmonics at 25 and $75 \mathrm{~Hz}$ are present and there is no sag, two "clean" spectral lines would appear. In the case of a hybrid event, the SK outlines the spectral leakage around the harmonic frequencies.

Finally, and with the two-fold purpose of illustrating harmonic detection and noise rejection at the same time, two pure harmonics (at 50 and $75 \mathrm{~Hz}$ ) have been contaminated by colored Gaussian noise $(\sigma=0.05$, bandwidth: 250 to $350 \mathrm{~Hz}$ ). The effect is the clear distinction of the two spectral lines along with the noise vanishing. In Figure 9, the spectral lines are outlined both in the second and in the fourth-order domain (SK); in the latter, noise is completely rejected, as expected by a higher-order spectrum. 

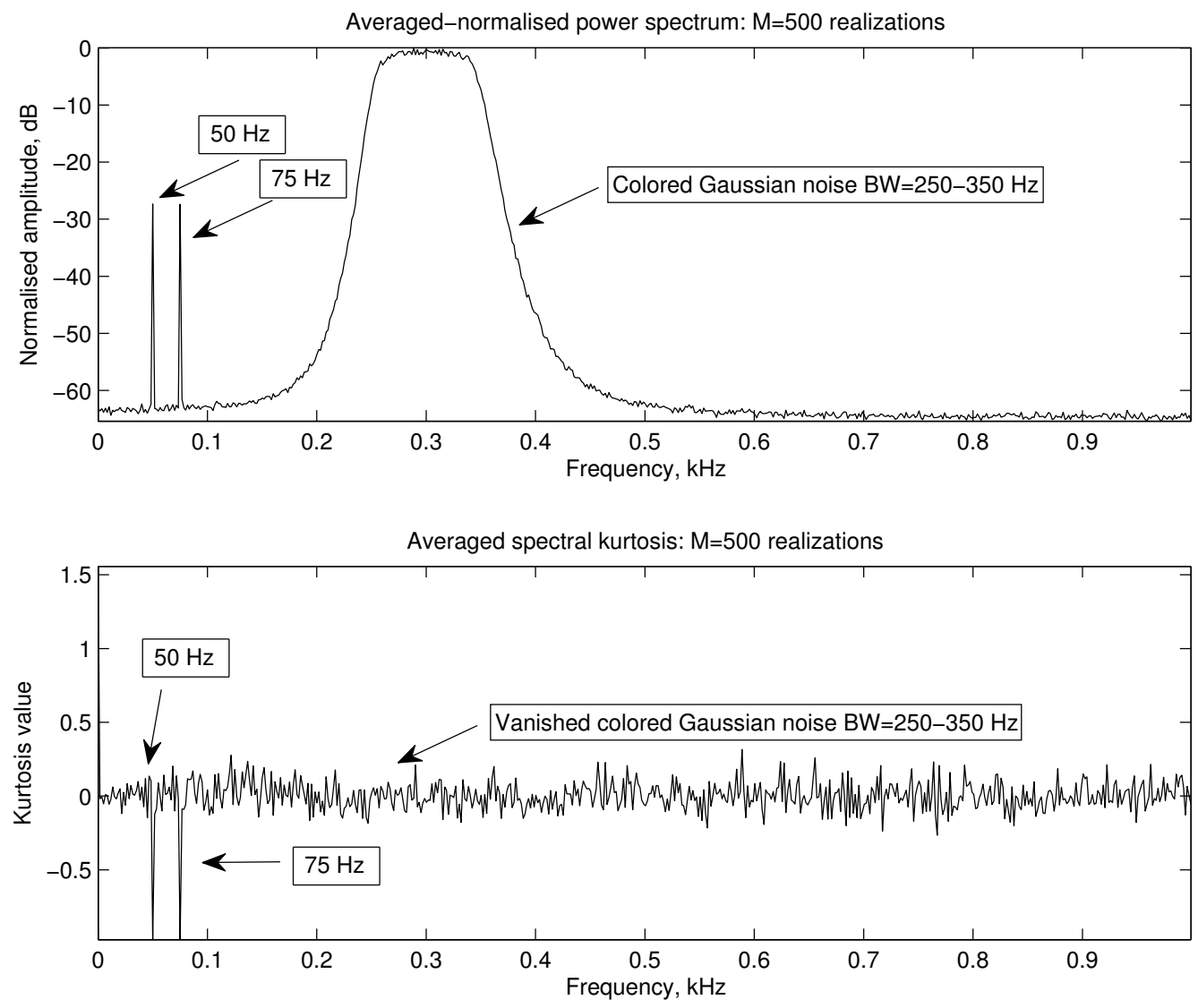

Figure 9. Harmonics' discrimination and noise rejection. Harmonics at 50 and $75 \mathrm{~Hz}$ have been mixed with colored Gaussian noise ( $\sigma=0.05$, bandwidth: 250 to $350 \mathrm{~Hz}$ ).

\section{Conclusions}

This paper shows that the SK has good noise resistance and is a robust spectral analysis tool, returning a similar response with different noise contamination levels (uniform and Gaussian noise produce the same results). As a variability analysis tool, associating the peakedness of the probability density function, and in the frame of PQ analysis, different sag depths have returned different kurtosis maximum values, but always with the same shape, showing the SK normal value at $150 \mathrm{~Hz}$. The sag length has no effect on the kurtosis response, showing similar values for each sag depth.

In the coupled disturbances study, sag conditions have been set constant in order to obtain similar test conditions. A frequency of $200 \mathrm{~Hz}$ has been introduced with oscillatory transients and harmonic distortion, returning a peak centered at the same position. Peaks related to these show a maximum width of $140 \mathrm{~Hz}$, which means a range from 130 to $270 \mathrm{~Hz}$. In the considered sag conditions, the maximum kurtosis value is 0.8 at $130 \mathrm{~Hz}$, with an average kurtosis value of 0.05 . That confirms that there is no interference from the sag SK response for the oscillatory transient and harmonic disturbance SK response.

Oscillatory transients show an exponential amplitude decay, so the alteration of the base wave is lower than in harmonic distortion, because this second situation keeps its amplitude constant. In addition, the oscillatory transient has one hard transition, at its start, because of its end amplitude being zero. On 
the other hand, harmonic disturbance have two hard transitions, one at its start and one at its end. All of these create higher values in harmonic disturbance situations, with even higher impact on the central frequency, for the same amplitude and length conditions. In addition, crisper peaks are obtained in harmonic distortion, due to a higher impact over the central frequency than in the oscillatory transient situation, in which adjacent frequencies are more affected. Taking all of this into consideration, when a symmetrical peak appears in the SK response, wide and soft ones are related to oscillatory transients and narrow and crisp ones to harmonic distortion.

As a final remark, the performance of the SK over hybrid (mixed) PQ events is promising, and it has been proven over a set of synthetics. The next step will focus the implementation on an autonomous measurement system, which requires adapting the measurement requirements to norms or the International Electrotechnical Commission (IEC). Compliance with the standards [27,28] that define the conditions for data acquisition in harmonic measurements for power supply systems implies the use of a basic acquisition window corresponding to a time period of $\Delta \mathrm{t}=200 \mathrm{~ms}$. Sampling frequency and the rest of the parameters have to be adapted to these requirements.

\section{Acknowledgments}

This work is funded and supported by the Spanish Ministry of Economy and Competitiveness in the frame of the Statal Plan for Excellency in Scientific and Technical Research, via Research Project TEC $2013-47316-C 3-2-P(S C E M S-A D-T E D-P Q R)$. In addition, the authors would like to thank the Andalusian Government for funding Research Unit PAIDI-TIC-168.

\section{Author Contributions}

Juan José González de la Rosa and José María Sierra-Fernández have arranged the electronic instruments and acquired the data series of electrical perturbations. Agustín Agüera Pérez, José Carlos Palomares Salas and Álvaro Jiménez-Montero have analyzed the time series from the measurements (feature extraction from the SK graphs).

\section{Conflicts of Interest}

The authors declare no conflict of interest.

\section{References}

1. Esmaili, M.; Rajabi, M. Optimal charging of plug-in electric vehicles observing power grid constraints. IET Gener. Transm. Distrib. 2014, 8, 583-590.

2. Clement-Nyns, K.; Haesen, E.; Driesen, J. The impact of Charging plug-in hybrid electric vehicles on a residential distribution grid. IEEE Trans. Power Syst. 2010, 25, 371-380.

3. Buzna, L.; Issacharoff, L.; Helbing, D. The evolution of the topology of high-voltage electricity networks. Int. J. Crit. Infrastruct. 2009, 5, 72-85.

4. Rohden, M.; Sorge, A.; Witthaut, D.; Timme, M. Impact of network topology on synchrony of oscillatory power grids. Chaos 2014, 24, 1-19. 
5. Pagani, G.; Aiello, M. Power grid complex network evolutions for the smart grid. Phys. A: Stat. Mech. Appl. 2014, 396, 248-266.

6. Blaabjerg, F.; Teodorescu, R.; Liserre, M.; Timbus, A. Overview of control and grid synchronization for distributed power generation systems. IEEE Trans. Ind. Electron. 2006, 53, 1398-1409.

7. International Electrotechnical Commission. IEC 61000-2-8-2002: Part 2-8: Environment-Voltage Dips and Short Interruptions on Public Electric Power Supply Systems with Statistical Measurement Results; International Electrotechnical Commission (IEC): Geneva, Switzerland, 2008.

8. IEEE. IEEE Recommended Practice for Monitoring Electric Power Quality; IEEE Std 1159-2009 (Revision of IEEE Std 1159-1995); IEEE: Piscataway, NJ, USA, 2009; c1-c81.

9. Ribeiro, M.; Marques, C.; Duque, C.; Cerqueira, A.; Pereira, J. Detection of disturbances in voltage signals for power quality analysis using HOS. Eurasip J. Adv. Signal Process. 2007, 2007, 177-177 .

10. Gonzalez de la Rosa, J.; Lloret, I.; Puntonet, C.; Piotrkowski, R.; Moreno, A. Higher-order spectra measurement techniques of termite emissions. A characterization framework. Measurement 2008, 41, 105-118.

11. Mansour, A.; Mesleh, R.; Aggoune, E.H. Blind estimation of statistical properties of non-stationary random variables. Eurasip J. Adv. Signal Process. 2014, 2014, 21.

12. Agueera-Perez, A.; Palomares-Salas, J.C.; Gonzalez de la Rosa, J.J.; Sierra-Fernandez, J.M.; Ayora-Sedeno, D.; Moreno-Munoz, A. Characterization of electrical sags and swells using higher-order statistical estimators. Measurement 2011, 44, 1453-1460.

13. Gonzalez de la Rosa, J.J.; Agueera-Perez, A.; Palomares-Salas, J.C.; Sierra-Fernandez, J.M.; Moreno-Munoz, A. A novel virtual instrument for power quality surveillance based in higher-order statistics and case-based reasoning. Measurement 2012, 45, 1824-1835.

14. Gonzalez de la Rosa, J.J.; Agueera-Perez, A.; Palomares-Salas, J.C.; Moreno-Munoz, A. Higher-order statistics: Discussion and interpretation. Measurement 2013, 46, 2816-2827.

15. Yin, J.; Wang, W.; Man, Z.; Khoo, S. Statistical modeling of gear vibration signals and its application to detecting and diagnosing gear faults. Inf. Sci. 2014, 259, 295-303.

16. Miyazaki, R.; Saruwatari, H.; Nakamura, S.; Shikano, K.; Kondo, K.; Blanchette, J.; Bouchard, M. Musical-noise-free blind speech extraction integrating microphone array and iterative spectral subtraction. Signal Process. 2014, 102, 226-239.

17. Salas, J.C.P.; De la Rosa, J.J.G.; Sierra-Fernández, J.M.; Agüera-Pérez, A. HOS network-based classification of power quality events via regression algorithms. Series: Advanced signal processing techniques and telecommunications network infrastructures for Smart Grid analysis, monitoring and management. EURASIP J. Adv. Signal Process. 2015, 49, 1-11.

18. De la Rosa, J.J.G.; Sierra-Fernández, J.M.; Agüera-Pérez, A.; Salas, J.C.P.; Moreno-Muñoz, A. An application of the spectral kurtosis to characterize power quality events. Electr. Power Energy Syst. 2013, 49, 386-398. 
19. De la Rosa, J.J.G.; Salas, J.C.P.; Agüera-Pérez, A.; Sierra-Fernández, J.M.; Moreno-Muñoz, A. A novel virtual instrument for power quality surveillance based in higher-order statistics and case-based reasoning. Measurement 2011, 44, 1453-1460.

20. Bollen, M.H.J.; Gu, I.Y.H.; Axelberg, P.G.V.; Styvaktakis, E. Classification of underlying causes of power quality disturbances: Deterministic versus statistical methods. EURASIP J. Adv. Signal Process. 2007, 2007, 1-17.

21. Gu, Y.H.; Bollen, M.H.J. Time-frequency and time-scale domain analysis of voltage disturbances. IEEE Trans. Power Deliv. 2000, 15, 1279-1283.

22. Ribeiro, M.V.; Marques, C.A.G.; Duque, C.A.; Cerqueira, A.S.; Pereira, J.L.R. Detection of disturbances in voltage signals for power quality analysis using HOS. EURASIP J. Adv. Signal Process. 2007, 2007, 1-13.

23. Ribeiro, M.V.; Pereira, J.L.R. Classification of single and multiple disturbances in electric signals. EURASIP J. Adv. Signal Process. 2007, 2007, 1-18.

24. Agüera-Pérez, A.; Palomares-Salas, J.C.; De la Rosa, J.J.G.; Sierra-Fernández, J.M.; Ayora-Sedeño, D.; Moreno-Muñoz, A. Characterization of electrical sags and swells using higher-order statistical estimators. Measurement 2011, 44, 1453-1460.

25. Gerek, O.N.; Ece, D.G. Power-quality event analysis using higher order cumulants and quadratic classifiers. IEEE Trans. Power Deliv. 2006, 21, 883-889.

26. Liu, Z.; Zhang, Q.; Han, Z.; Chen, G. A new classification method for transient power quality combining spectral kurtosis with neural network. Neurocomputing 2014, 125, 95-101.

27. International Electrotechnical Commission. IEC 61000-4-7: Testing and Measurement Techniques. General Guide on Harmonics and Interharmonics Measurements and Instrumentation, for Power Supply Systems and Equipment Connected Thereto; International Electrotechnical Commission (IEC): Geneva, Switzerland, 2002.

28. International Electrotechnical Commission. IEC 61000-4-30: Testing and Measurement Techniques. Power Quality Measurement Methods; International Electrotechnical Commission (IEC): Geneva, Switzerland, 2008.

(c) 2015 by the authors; licensee MDPI, Basel, Switzerland. This article is an open access article distributed under the terms and conditions of the Creative Commons Attribution license (http://creativecommons.org/licenses/by/4.0/). 